\title{
Patient Preferences for Receiving Reports of Test Results
}

\author{
Jeannine R. LaRocque, PhD, Christina L. Davis, BS, Tina P. Tan, BA, \\ Frank J. D'Amico, PhD, and Daniel J. Merenstein, MD
}

Introduction: The general public's preferences for modes of communication (other than in-person communication) for medical test results were investigated. We hypothesized that patients would prefer a variety of methods to receive common tests results (blood cholesterol and colonoscopy) compared with genetics test results.

Methods: This study was a cross-sectional survey.

Results: A total of 409 participants responded to the survey. Among these participants, $\geq 50 \%$ reported that they were comfortable receiving results for a blood cholesterol test or colonoscopy via 4 of the 7 non-in-person communication methods (password-protected website, personal voicemail, personal E-mail, and letter were preferred over home voicemail, fax, and mobile phone text message). In comparison, $>50 \%$ of participants were comfortable with only 1 non-in-person communication method for non-HIV sexually transmitted infections (STIs) and none for genetic tests. Patients were least comfortable receiving any information via fax, regardless of test type. There were statistical differences among comfort levels for blood cholesterol and colonoscopy tests and both STIs and genetic testing for personal voicemail, personal E-mail, mobile phone text message, and password-protected website, but there were no differences between STIs and genetic testing. No correlation was found between "familiarity" with test and "comfort" of receiving information about specific test.

Conclusions: Participants demonstrated preferences in how they received test results by non-inperson communication methods, preferring personal E-mail and password-protected websites, but did not prefer fax. Importantly, participants also demonstrated that preference was dependent on test type. (J Am Board Fam Med 2015;28:759-766.)

Keywords: Communication, Doctor-Patient Relations, Electronic Medical Records, Medical Laboratory Science, Patient-Centered Care, Patient Preference

Effective patient-physician interactions depend on trust, suitable communication, and appropriate, timely interventions. Proper delivery of primary

This article was externally peer reviewed.

Submitted 15 January 2015; revised 2 June 2015; accepted 8 June 2015.

From the Department of Human Science, Georgetown University Medical Center, Washington, DC (JRL, CLD, DJM); the Department of Family Medicine, Georgetown University Medical Center, Washington, DC (TPT, DJM); the Department of Mathematics and Computer Science, Duquesne University, Pittsburgh, PA (FJD); and the St. Margaret Family Medicine Residency Program, University of Pittsburgh Medical Center, Pittsburgh, PA (FJD).

Funding: none.

Conflict of interest: none declared.

Corresponding author: Jeannine LaRocque, $\mathrm{PhD}$, School of Nursing and Health Studies, 265 St. Mary's Hall, 3700 Reservoir Rd. NW, Washington, DC 20057 (E-mail: jan. larocque@georgetown.edu). health care information is the foundation of this success. Patients undergo medical tests for a wide variety of reasons, including diagnosing a disease or determining the likelihood of developing a disease. ${ }^{1}$ The results from tests range from simple and straightforward, such as blood cholesterol levels, to potentially more complex and sensitive, such as inheriting a mutation that leads to a predisposition to a genetic disorder. It is necessary for health professionals to deliver results using the most confidential and patient-oriented method possible. Despite these needs, no standardized delivery method has been established, nor have preferences been clearly delineated. ${ }^{2}$

The availability of various and newer forms of communication permits delivery of information in a variety of ways, some of which physicians or 
patients may prefer. Older research has shown that patients are apprehensive about using E-mail because of privacy concerns. ${ }^{3}$ However, this is an older study conducted when most patients' access to E-mail was limited to the work environment. It has been demonstrated that patients from a wide range of socioeconomic backgrounds support shared medical records. Furthermore, a randomized controlled trial showed patients given access to portals demonstrated increased satisfaction., ${ }^{4,5}$ Given concerns with the privacy of health information, Congress addressed the importance of protecting the privacy of personal health information by developing the Health Insurance Portability and Accountability Act (HIPPA) in 1996 (reviewed in ref. ${ }^{6}$ ). Since the passage of HIPPA, however, there has been an expansion to new Internet-based methods, such as E-mail and password-protected websites, and the potential use of emerging technologies such as mobile text messages (reviewed in ref. ${ }^{7}$ ). Interestingly, the Department of Health and Human Services now requires laboratories to provide to patients direct access to results and suggests, but does not require the delivery of test results through patient portals or personal health records. ${ }^{8}$

Reported preferences for these methods vary; some studies suggest US mail as a preference, whereas others reflect a preference for telephone communication..$^{9-11}$ These studies report a lack of preference in electronic methods of delivering test results, stressing a concern over the security of information with this form of communication. ${ }^{10,11}$ However, technology has advanced remarkably, and consumers are more familiar with working with private information over the Internet.

This study was conducted to gain greater insight into the general public's preferences to modes of communication between them and their health care provider with regard to specific medical test results. Understanding these factors could greatly improve patient satisfaction while minimizing suboptimal patient care within the primary health care system. We hypothesized that there would be differences in preferences of method of delivery of test results, and both the method and the type of test itself will affect these preferences. Specifically, we believed that participants would be most comfortable receiving the results of common tests, such as blood cholesterol and colonoscopy, in a variety of ways but would be more cautious about how they re- ceived information regarding sexually transmitted infections (STIs) and genetic diseases.

\section{Methods}

\section{Study Design and Participants}

A cross-sectional sample survey was conducted. The Georgetown University Institutional Review Board approved this study, and all participants gave implied informed consent.

The survey included questions on patient demographics, familiarity with certain medical tests, and patient preferences for delivery of medical test results from their health care provider. Self-described knowledge of their comfort level for certain medical tests was obtained. In addition, comfort levels with certain delivery methods of medical test results were also collected, defined on a Likert scale from 1 to 10 , where 1 represents "not comfortable at all" and 10 represents "very comfortable." Six specific medical tests were described: cholesterol levels, colonoscopy, a test for a non-human immunodeficiency virus STI, and 3 genetic tests (predisposition to genetic mutations, inherited genetic mutations, and carrier of genetic mutations). The 7 delivery methods were fax, personal voicemail, home voicemail, personal E-mail, letter, mobile phone text message, and password-protected website.

The survey was administered electronically and by Article, through intercept surveying on the Georgetown University campus. It was electronically administered through SurveyMonkey (www. surveymonkey.com). The URL to the survey was made available on a public invitation page on $\mathrm{Fa}$ cebook (www.facebook.com) and distributed through personal E-mail. Any person 18 years of age and older who was able to read the survey (in English) was eligible to participate. No compensation was provided to participants.

\section{Data Analysis}

All survey data were compiled in Excel (Microsoft Office; Microsoft Corp., Redmond, WA) and screened for completion and accuracy. Few questions were not answered, and thus no imputed values for missing data were required for statistical analysis. Age was grouped (18 to 24,25 to 34,35 to 54 , $\geq 55$ years) for statistical comparisons. Before examining data, it was decided that Likert scale responses of the comfort levels would be classified 
into 3 categories: "not comfortable" (score 1 to 3), "ambivalent" (score 4 to 7), and "comfortable" (score 8 to 10). Percentages of each category were determined for descriptive comparisons. Pearson correlations were used to calculate the strength of the linear relationships between the demographic question, "How familiar are you with blood tests for cholesterol levels?" and the "cholesterol" response from the common test type. Similarly, the correlation between "How familiar are you about genetic testing?" with the average across the 3 genetics areas was calculated.

To test differences in the comfort levels between the types, the 2 blood cholesterol and colonoscopy tests were averaged and the 3 tests within the genetics type were averaged. STI had only 1 question and it was used alone. Since participants answered questions for each of the 3 types of tests (common tests, STI, and genetic), their responses were correlated, and no loss of information or bias was observed after averaging the tests within the common and genetic types. Repeated-measures analysis of variance methods were used to compare the means between the 3 types. In the repeated measures tests, an exchangeable correlation matrix was incorporated in the estimation of the standard errors. Because of the multiplicity in testing the various combinations of types (common tests vs STI, STI vs genetic, and common tests vs genetic), $P$ values were adjusted for multiple comparisons $(P<$ .05) using Tukey's method.

Descriptive statistics and frequency tables were calculated using SPSS version 22.0 (IBM Corp., Armonk, NY), whereas the inferential statistical tests were done using SAS version 9 (SAS Institute, Cary, NC).

\section{Results}

A total of 409 participants responded to the survey, of whom 255 were women. Regarding surveys, 324 were completed through SurveyMonkey and 85 were completed on Article through local intercept surveying. The average age of participants was 37 years old, with the majority $(88 \%)$ being white. Table 1 shows the full demographic distribution of participants.

\section{Comfort Level of Receiving Test Results by Method}

The tests responses were grouped into 3 categories: common test results (cholesterol and colonoscopy),
Table 1. Demographic Characteristics of Survey

\section{Respondents}

Respondents $\left(^{*}\right)$

Sex $(\mathrm{n}=407)$
Female
Male
Age, years $(\mathrm{n}=406)$
Mean $(\mathrm{SD})$
$18-24$
$25-34$
$35-54$
$\geq 55$
Race $(\mathrm{n}=409)$
White
Asian
African American
Pacific Islander
American Indian
Other
Highest level of education $(\mathrm{n}=406)$
Did not complete high school
High school diploma
Bachelor's degree
Master's degree
Doctorate
Other

255 (62.7)

$152(37.3)$

$37.4(16.7)$

133 (32.8)

94 (23.2)

94 (23.2)

85 (20.9)

$362(88.5)$

17 (4.2)

14 (3.4)

$3(0.7)$

$1(0.2)$

12 (2.9)

$2(0.5)$

77 (19.0)

157 (38.7)

$82(20.0)$

77 (19.0)

$11(2.7)$

Do you have a usual source of care (a doctor's office, clinic, health center, or other place you go if you are sick or need advice about personal health matters)? $(\mathrm{n}=406)$

Yes

$375(92.4)$

No

$31(7.6)$

Have you or a family member been diagnosed with an inherited disease with a known genetic cause (for example, Down syndrome, cystic fibrosis, sickle cell anemia)? $(\mathrm{n}=405)$

No.

$335(82.7)$

Yes, I have.

$14(3.5)$

$48(11.9)$

$8(2.0)$

Yes, both myself and a family member.

$6.5(3.3)$

a scale of 1 to $10, \dagger$ how familiar are you
with blood tests for cholesterol levels? $(\mathrm{n}=405)$, mean (SD)

On a scale of 1 to $10, \dagger$ how familiar are you about genetic testing? $(n=404)$, mean (SD)

Data are $\mathrm{n}(\%)$ unless otherwise indicated.

${ }^{*}$ Missing values were not included.

†On a scale of 1 to $10(1=$ not familiar at all; $10=$ very familiar). SD, standard deviation.

STIs, and genetic tests (predisposed, inherited, and carrier). For the purposes of comparison, Figure 1 shows the percentages observed for the "comfortable," "ambivalent," and "not comfortable" classi- 
Figure 1. Comfort with receiving test results by method of communication. Each method of test result delivery (7 total) and type of test ( 3 total) is given. Participants' comfort level with various tests and methods are presented. Those scoring 8 to 10 on the Likert scale were grouped as "comfortable." Those scoring 4 to 7 on the Likert scale were grouped as "ambivalent." Those scoring 1 to 3 on Likert scale were grouped as "not comfortable." For each type of test, the blood cholesterol and colonoscopy tests were grouped as "common tests" and the 3 genetics tests were grouped as "genetics" (see Methods for details). HIV, human immunodeficiency virus; STI, sexually transmitted infection.

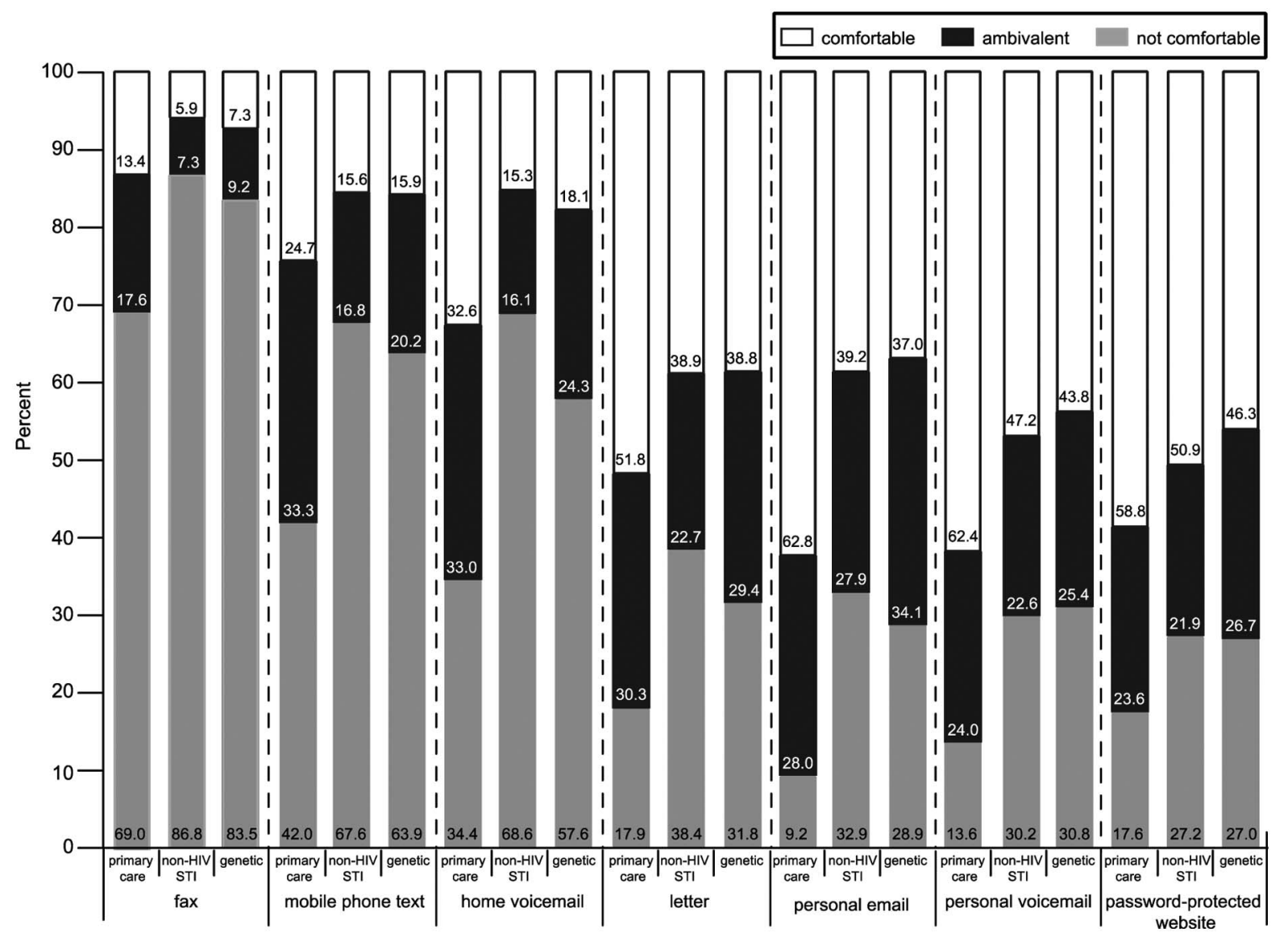

fications for each method of communication. This figure shows that participants were, by a wide margin, least comfortable receiving any information via fax: $13.4 \%$ were comfortable receiving common test results via fax, compared with $5.9 \%$ for STI and $7.3 \%$ for genetic tests. This was followed by participants not comfortable receiving information via home voicemail: $32.6 \%$ were comfortable receiving the common test results, compared with only $15.3 \%$ for STI and $18.1 \%$ for genetic tests. This is in contrast to personal voicemail, through which participants were comfortable receiving information: $62.4 \%$ were comfortable receiving blood cholesterol test and colonoscopy results via personal voicemail, compared with $47.2 \%$ for STI and $43.8 \%$ for genetic tests. This higher level of comfort was similar to that of receiving results via a password-protected website: common tests, $58.8 \%$; STI tests, $50.9 \%$; and genetic tests, $46.3 \%$. In 4 of the 7 communication categories, participants reported $\geq 50 \%$ comfort levels for different methods of receiving information for a common test, whereas, in comparison, $>50 \%$ of patients were comfortable with only 1 method for STI (password-protected website; $50.9 \%$ ) and none for genetic tests.

\section{Mean Differences in Comfort Level}

Mean responses in comfort levels for the 3 types of tests were examined and statistically compared ( $\mathrm{Ta}$ ble 2). Table 2 shows that, for every method of communication, some if not all combinations were statistically different. However, even though some 
Table 2. Responses* from Questionnaire Items on How Comfortable Respondents Were With Receiving Specific Tests Results via the Listed Methods of Communication from a Health Care Provider

\begin{tabular}{|c|c|c|c|c|c|c|c|}
\hline \multirow[b]{3}{*}{ Method of Communication } & \multicolumn{6}{|c|}{ Type of Test } & \multirow{3}{*}{$\begin{array}{c}P \\
\text { Valuet† }\end{array}$} \\
\hline & \multicolumn{2}{|c|}{ Common Tests (Group 1) } & \multirow{2}{*}{ 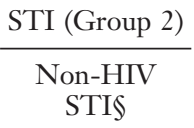 } & \multicolumn{3}{|c|}{ Genetic Test (Group 3) } & \\
\hline & Cholesterol $†$ & Colonoscopy $\ddagger$ & & Predisposed $\|$ & Inherited $\mathbb{1}$ & Carrier $^{* *}$ & \\
\hline Fax & 3.5 & 2.6 & 1.9 & 2.2 & 2.0 & 2.1 & All \\
\hline Personal voicemail & 7.8 & 6.9 & 6.2 & 6.5 & 5.8 & 6.0 & $\begin{array}{l}1 \text { vs } 3 ; 1 \\
\text { vs } 2\end{array}$ \\
\hline Home voicemail & 5.8 & 4.7 & 3.2 & 4.2 & 3.6 & 3.8 & All \\
\hline Personal E-mail & 8.0 & 7.2 & 5.7 & 6.2 & 5.5 & 5.8 & $\begin{array}{c}1 \text { vs } 3 ; 1 \\
\text { vs } 2\end{array}$ \\
\hline Letter & 7.2 & 6.4 & 5.5 & 6.1 & 5.6 & 5.8 & All \\
\hline Mobile phone text message & 5.2 & 4.1 & 3.2 & 3.6 & 3.3 & 3.3 & $\begin{array}{c}1 \text { vs } 3 ; 1 \\
\text { vs } 2\end{array}$ \\
\hline Password-protected website & 7.5 & 6.9 & 6.6 & 6.6 & 6.1 & 6.5 & $\begin{array}{c}1 \text { vs } 3 ; 1 \\
\text { vs } 2\end{array}$ \\
\hline
\end{tabular}

Data are shown as the mean values.

*The response scale was based on 1 (not comfortable at all) to 10 (very comfortable). Response numbers are approximately 409 within each cell; standard deviations ranged between 2.2 and 3.7.

†You received a test to check the level of cholesterol in your blood.

$\ddagger$ You received a colonoscopy to check for the potential development of colon cancer.

\$You received a test to determine whether you have contracted a non-HIV sexually transmitted infection (STI; for example, herpes).

$\|$ You received a test to determine whether you are genetically predisposed to a certain disease (you could potentially develop the disease, but it is not guaranteed).

IY You received a test to determine whether you have inherited a genetic disease that is currently untreatable.

${ }^{* *}$ You received a test to determine whether you are a carrier for a mutation that causes a genetic disease that could be passed on to your children.

$\dagger \uparrow P$ value for testing the means of the individual tests within each type (common tests, 1 ; STI, 2; and genetic, 3 ). "All” implies groups 1 vs 2,1 vs 3 , and 2 vs 3 are significantly different. The $P$ values were adjusted for multiple comparisons $(P<.05)$.

HIV, human immunodeficiency virus.

differences in the means were not clinically large, important patterns are still worth noting. For instance, for the fax method of communication, the mean comfort scores were relatively low (1.93 to 3.52) across all the questions. Consistent with our hypothesis for personal voicemail, personal E-mail, mobile phone text message, and password-protected website, there were statistical differences among common tests and both STI and genetic testing, but not between STI and genetic testing.

Further, some methods of communication were related to age. For example, $71 \%$ of participants over age 55 were comfortable receiving a letter for a common test result compared with only $35 \%$ of participants ages 18 to 24 years. Surprisingly, however, we did not observe differences with technology, as personal E-mail and password-protected website preferences were not affected by age (data not shown).

\section{Knowledge and Method Preference Association}

When examining the relationship between "familiarity" with blood tests and "comfort" with receiv- ing information via different methods, in all cases there was little to no correlation. For cholesterol testing, the largest $r$ correlation coefficient was 0.21 , similar to genetic testing, for which the largest $r$ correlation coefficient was 0.14 . The distributions of responses were symmetric; there were no outliers or gaps in the graphs to help account for the low correlations.

\section{Discussion}

This study was conducted to determine whether patients have a preference in the method of delivery of specific test results. Regardless of the type of test, we found that the majority of participants were not comfortable with delivery via fax. Although no method was preferred by a majority of participants regardless of the test, the largest portion of participants were comfortable receiving test results through password-protected websites. This is consistent with past research that demonstrated that, for those who enrolled in patient portals, messaging systems 
were preferred over phone calls. ${ }^{12}$ More recent research indicates that patient enrollment in password-protected websites (patient portals) ranges from $40 \%$ to $69 \%$, although this varies depending on medical practice and is often associated with racial/ethnic disparities. ${ }^{13-15}$ Considering these studies, it is not surprising that password-protected websites was the method most preferred by participants in this study. However, much of the information about patient portal use has been disseminated via conferences and informal means, and has not been extensively studied in the published literature. In addition, patient portal use is encouraged to fulfill the requirement by the Department of Health and Human Services for direct access of patients to their test results. ${ }^{8}$ There are concerns that providing patients with this direct information may result in patients misinterpreting results; however, our data show that patients prefer patient portals, even for highly sensitive information such as STI and genetic results. ${ }^{16}$

We also found that the type of test affects the overall comfort level with methods of delivery. This may be because of the range of types of results and subsequent follow-up treatment. How people perceive a specific illness or condition, the potential treatments for each condition, and adherence to these treatment plans could affect their preferred method of delivery for the results of specific tests. ${ }^{17}$ This may be especially true in genetic testing, since there is a widespread belief that genetic conditions are not treatable. ${ }^{17}$

As hypothesized, the preferences of methods for delivery of non-HIV STI test results were more similar to those of genetic tests than blood cholesterol and colonoscopy tests. While most participants were comfortable with a variety of methods to deliver blood cholesterol and colonoscopy tests (participants reported $\geq 50 \%$ comfort levels for 4 of 7 communication categories), only with passwordprotected website were a majority of participants, at $50.9 \%$ for STI, comfortable receiving results. As previously discussed, the most preferred method for both of these test types was password-protected websites. Both STI and genetic test results have potential implications for the individual patients as well as related family members; as such, there may be a difference in preference for the method of communicating these results. While studies suggest that results from a cancer susceptibility tests have the same impact on patients whether they are de- livered in person or over the telephone, this is, to our knowledge, the first study to date that has addressed the preference of communication methods that are not in-person. ${ }^{18,19}$ In addition, related to the sensitive nature of specific types of tests is the impact of patients' trust in the confidentiality of the information. Studies reporting a preference for US mail or telephone call reflect patients' perceptions that these are the most secure forms of communication. ${ }^{11}$ More sensitive test results could therefore affect the degree to which patients are concerned with confidentiality. This study suggests that patients may prefer the option to choose the method of delivery of medical test results, depending on the type of test administered.

The increasing use of electronic methods of delivery and the familiarity of different patient populations with emerging technologies is likely affected by age. Previous research has shown that young patients preferred electronic mail as a delivery method significantly more than older patients, who preferred US mail as the first choice of method of notification. ${ }^{9} \mathrm{We}$ also observed an increase in comfort with receiving test results via letter as the age of the patient increased. However, personal voicemail preferences decreased with age, and we observed a limited change in the preference for methods requiring computer access, such as personal E-mail and password-protected website.

Patient education of the specific field or test result may influence their preference for the method of test result delivery. A qualitative study of preferences for obtaining mammogram results among urban women found that many of the women had difficulty interpreting the results in a typical results notification letter. ${ }^{20}$ Many women misinterpreted their results because they could not understand the language their providers used in the letter. However, we did not observe a significant correlation between a patient's self-described understanding of a test and their comfort level with the delivery of test results. This is important information because it suggests that, regardless of a patient's familiarity with the medical testing process, patients are consistent in their comfort levels with how the results are delivered. A recent report showing that patient education regarding the importance of colorectal cancer screening for at-risk patients had a minimal effect on overall screening rates supports our findings. ${ }^{21}$ 
Limitations of this study include that we did not observe physicians and patients in practice but queried participants' perceived preferences. Our approach does give valuable ideal information for physicians to consider when relaying information, but practice patterns and resources also dictate how information is provided. We are unable to provide a survey response rate because of the survey distribution method. While a portion of respondents completed the survey through Article (85 of 409), a large proportion of surveys were completed through Facebook and E-mail (324 of 409). Those respondents who completed the survey electronically may be more comfortable with electronic test delivery methods than the overall patient population. In addition, our sample population is representative of many health care provider offices in the United States, but, clearly, different ethnicities, income levels, education levels, language preferences, and other variables may change preferences.

Results reported here contribute to the growing body of work aiming to improve patient-physician communication. While there are many communication opportunities in a patient-physician relationship, the preference of how medical test results are delivered has not been extensively analyzed. Specifically, both the type of test results and different means of communication need to be considered. Our study suggests that this is indeed the case, and that the ideal method of delivery of test results differs depending on the context and sensitivity of the test itself. Importantly, it seems that for common results such as colonoscopy and cholesterol, there are many means through which patients are comfortable receiving information. For more sensitive tests, however, such as STI and genetic test results, physicians need to consider using password-protected websites. Further research should attempt to examine these issues in real time, as patients are receiving information, to better understand preferences.

\section{References}

1. Hassol A, Walker JM, Kidder D, et al. Patient experiences and attitudes about access to a patient electronic health care record and linked web messaging. J Am Med Inform Assoc 2004;11:505-13.

2. Boohaker EA, Ward RE, Uman JE, McCarthy BD. Patient notification and follow-up of abnormal test results. A physician survey. Arch Intern Med 1996; 156:327-31.

3. Fridsma DB, Ford P, Altman R. A survey of patient access to electronic mail: attitudes, barriers, and opportunities. Proc Annu Symp Comput Appl Med Care 1994;15-9.

4. Ross SE, Todd J, Moore LA, Beaty BL, Wittevrongel L, Lin CT. Expectations of patients and physicians regarding patient-accessible medical records. J Med Internet Res 2005;7:e13.

5. Fowles JB, Kind AC, Craft C, Kind EA, Mandel JL, Adlis S. Patients' interest in reading their medical record: relation with clinical and sociodemographic characteristics and patients' approach to health care. Arch Intern Med 2004;164:793-800.

6. Moskop JC, Marco CA, Larkin GL, Geiderman JM, Derse AR. From hippocrates to HIPAA: privacy and confidentiality in emergency medicine-Part I: conceptual, moral, and legal foundations. Ann Emerg Med 2005;45:53-9.

7. Fortney JC, Burgess JF Jr, Bosworth HB, Booth BM, Kaboli PJ. A re-conceptualization of access for 21 st century healthcare. J Gen Intern Med 2011;26(Suppl 2):639-47.

8. Centers for Medicare \& Medicaid Services (CMS), HHS; Centers for Disease Control and Prevention (CDC), HHS; Office for Civil Rights (OCR), HHS. CLIA program and HIPAA privacy rule; patients' access to test reports. Final rule. Fed Regist 2014;79: 7289-316.

9. Grimes GC, Reis MD, Budati G, Gupta M, Forjuoh SN. Patient preferences and physician practices for laboratory test results notification. J Am Board Fam Med 2009;22:670-6.

10. LaVela SL, Schectman G, Gering J, Locatelli SM, Gawron A, Weaver FM. Understanding health care communication preferences of veteran primary care users. Patient Educ Couns 2012;88:420-6.

11. Baldwin DM, Quintela J, Duclos C, Staton EW, Pace WD. Patient preferences for notification of normal laboratory test results: a report from the ASIPS collaborative. BMC Fam Pract 2005; 6:11.

12. Liederman EM, Morefield CS. Web messaging: a new tool for patient-physician communication. J Am Med Inform Assoc 2003;10:260-70.

13. Yamin CK, Emani S, Williams DH, et al. The digital divide in adoption and use of a personal health record. Arch Intern Med 2011;171:568-74.

14. Ralston JD, Coleman K, Reid RJ, Handley MR, Larson EB. Patient experience should be part of meaningful-use criteria. Health Aff (Millwood) 2010;29:607-13.

15. Goel MS, Brown TL, Williams A, Hasnain-Wynia $\mathrm{R}$, Thompson JA, Baker DW. Disparities in enrollment and use of an electronic patient portal. J Gen Intern Med 2011;26:1112-6.

16. Young MJ, Scheinberg E, Bursztajn H. Direct-topatient laboratory test reporting: balancing access with effective clinical communication. JAMA 2014; 312:127-8. 
17. Langston AL, Johnston M, Francis J, et al. Protocol for stage 2 of the GaP study (genetic testing acceptability for Paget's disease of bone): a questionnaire study to investigate whether relatives of people with Paget's disease would accept genetic testing and preventive treatment if they were available. BMC Health Serv Res 2008;8:116.

18. Jenkins J, Calzone KA, Dimond E, et al. Randomized comparison of phone versus in-person BRCA1/2 predisposition genetic test result disclosure counseling. Genet Med 2007;9:487-95.

19. Platten U, Rantala J, Lindblom A, Brandberg Y, Lindgren G, Arver B. The use of telephone in ge- netic counseling versus in-person counseling: a randomized study on counselees' outcome. Fam Cancer 2012;11:371-9.

20. Marcus EN, Drummond D, Dietz N. Urban women's preferences for learning of their mammogram result: a qualitative study. J Cancer Educ 2012;27: $156-64$

21. Weinberg DS, Myers RE, Keenan E, et al. Genetic and environmental risk assessment and colorectal cancer screening in an average-risk population: a randomized trial. Ann Intern Med 2014;161: 537-45. 